\title{
Modelagem do Crescimento Populacional do Rebanho Bovino Brasileiro
}

\author{
Alfredo Ribeiro de Freitas ${ }^{1}$, Selene Maria Coelho Loibel ${ }^{2}$, Marinho Gomes de Andrade ${ }^{3}$, João \\ Bosco Ribeiro do $\mathrm{Val}^{4}$
}

\begin{abstract}
RESUMO - Considerando-se o número efetivo de animais e a taxa de abate do rebanho bovino brasileiro no período de 1983 a 2000 , estimou-se o crescimento dessa população utilizando-se o modelo de Richards, ajustado pela técnica de verossimilhança profile. O modelo se mostrou adequado para descrever o crescimento da população brasileira de bovinos, pois as superestimavas e ou subestimavas dos valores se situaram entre 1 e 2,5\%. A partir da modelagem por cadeia de Markov, foram calculados a probabilidade de o rebanho atingir 200 milhões de animais até o ano de 2015, em função da taxa de abate, e o tempo esperado para se atingir este tamanho populacional, em função da taxa de abate. A probabilidade de o rebanho atingir 200 milhões de animais até o ano de 2015, a uma taxa de abate de aproximadamente $17 \%$, é 0,7 . Com taxa de abate anual de 16\%, o rebanho atingirá esse tamanho no período de 11 anos e, com taxa de abate de $18 \%$, em 20 anos.
\end{abstract}

Palavras-chave: cadeias de Markov, função de verossimilhança profile, modelo de crescimento de Richards, indicadores de viabilidade da população

\section{Modeling the Growth of Brazilian Cattle Population}

\begin{abstract}
The growth of the Brazilian bovine cattle population was evaluated using the effective number of animals and the annual slaughter rate from 1983 to 2000. The Richards model was fitted with the profile likelihood technique. Two population parameters were calculated by Markov chain modeling: a) the probability of the cattle population to reach 200 million of animals in 2015 as a function of the slaughter rate; b) the time to reach this size, considering different annual slaughter rates. The Richards model was adequate to estimate the Brazilian cattle population growth since overestimated and/or underestimated values ranged between one and 2.5\%. The probability of the Brazilian herd to reach 200 millions animals in 2015 for an annual slaughter rate of approximately $17 \%$ is 0.7 and the expected time to reach 200 million animals for annual slaughter rates of $16 \%$ and $18 \%$ was 11 and 20 years respectively.
\end{abstract}

Key Words: Markov Chain, population growth indicators, profile likelihood function, Richards growth model

\section{Introdução}

Enquanto a população bovina mundial tende a estabilizar, ou mesmo reduzir, como acontece nos grandes países produtores de carne (Pineda, 2000), o rebanho brasileiro aumentado nos últimos anos, possuindo atualmente em torno de 164 milhões de animais, com crescimento de 10 milhões apenas nos últimos sete anos (ANUALPEC, 2002).

No novo milênio, o Brasil possui o segundo maior rebanho bovino, de modo que sua produção de carne bovina é superada apenas pelos Estados Unidos (ANUALPEC, 2002). A bovinocultura de corte tem grande potencial para continuar em crescimento, sendo favorecida por vários fatores, desde sua dimensão territorial, com grandes propriedades em regiões de terras produtivas, como no Centro-Oeste, e suas condições climáticas até sua composição genética, com predominância de aproximadamente $80 \%$ de animais de origem zebuína. Esta combinação, associada às tecnologias modernas de melhoramento genético animal, de eficiência do manejo e da sanidade, entre outras, tem possibilitado a exploração de uma pecuária ecológica, relativamente extensiva, com pastagens predominantemente naturais e de baixo custo.

O desenvolvimento da bovinocultura de corte torna-se ainda mais atraente quando se considera o potencial futuro desse setor no país. Estima-se que nos próximos dez anos as exportações brasileiras de carne bovina crescerão $170 \%$ em quantidade e $250 \%$ em valor, passando das 930 mil toneladas de equivalente carcaça em 2002 para aproximadamente 2,5 milhões de toneladas em 2012. Nesse mesmo período, a exportação passará de US\$1,5 bilhão para US\$ 4,5

\footnotetext{
${ }^{1}$ Pesquisador da Embrapa Pecuária Sudeste, Caixa Postal 339, 13560-970 - São Carlos, SP. Bolsista do CNPq. Email: ribeiro@cppse.embrapa.br

2 Doutora, DT-FEE-UNICAMP.

3 Professor Doutor, Instituto de Ciências Matemáticas de São Carlos - ICMC/USP.

4 Professor Doutor, DT-FEE-UNICAMP.
} 
bilhões anuais (ANUALPEC, 2003). Segundo Pineda (2000), uma estratégia para o Brasil aproveitar todas as suas potencialidades e se consolidar definitivamente no cenário internacional como grande produtor e exportador de carne bovina seria a manutenção de uma população bovina de aproximadamente 200 milhões de animais.

No caso da produção bovina brasileira, entre os fatores responsáveis pelas variações aleatórias associadas ao crescimento, encontram-se os sistemas de criação, que normalmente são desenvolvidos em regime de pastagens extensivas, em que os animais são sujeitos à escassez periódica de forragem, que compromete o seu desenvolvimento e sua eficiência reprodutiva, e a falta de adequação do potencial genético dos rebanhos ao ambiente e ao manejo, ou vice-versa.

Portanto, é importante o uso de metodologias que possibilitem estimar com confiabilidade o crescimento da população bovina no país, pois possibilitaria a organização política do consumo interno, de exportação e de marketing, entre outros. Existem vários modelos utilizados para estimação do crescimento populacional de animais; um deles é o modelo de Richards (Richards, 1959) - apresentado como generalização dos modelos logístico e Gompertz (Fitzhugh, 1976), que, além de descrever o crescimento populacional, tem sido bastante utilizado para cálculo das curvas de crescimento em animais e para análises de medidas repetidas ou de dados longitudinais (Fitzhugh, 1976; Paz, 2002). Neste caso, os parâmetros possuem interpretabilidade biológica e resumem de forma simplificada as características de crescimento da população.

Em razão de algumas particularidades do modelo de Richards para estimativa do crescimento populacional, como a não-linearidade, algumas alternativas computacionais são requeridas para o seu uso, como a função de verossimilhança profile, utilizada no cálculo das estimativas clássicas (BarndorffNielsen \& Cox, 1994), que permite estimar os parâmetros eliminando-se os problemas numéricos da função de verosimilhança clássica.

O modelo de Richards também tem mostrado sua versatilidade para o estudo de crescimento populacional em outras espécies. Hammerle et al. (2002), por exemplo, compararam os modelos de Gompertz, Richards e Verhulst entre si para estimar o crescimento populacional de Escherichia coli e concluíram que todos proporcionaram bons resultados. A função de Richards tem sido empregada em estudos de curvas de crescimento de várias espécies animais, como frangos de corte (Freitas et al., 1984) e bovinos (Perotto et al., 1992; Paz, 2002).

O objetivo neste trabalho foi estimar o crescimento da população brasileira de bovinos pelo modelo de Richards considerando-se o número efetivo de animais e a taxa de abate do rebanho no período de 1983 a 2000 (ANUALPEC, 2001), visando obter a probabilidade de o rebanho atingir 200 milhões de animais até o ano de 2015, com taxa de abate de 19 a 20\%, e o tempo para se atingir 200 milhões de animais com taxa de abate anual de 19 a $20 \%$.

\section{Material e Métodos}

Foram utilizados o número efetivo de animais e a taxa anual de abate anual do rebanho bovino brasileiro no período de 1983 a 2000 (Tabela 1) para se estimar seu crescimento populacional pelo modelo de Richards.

A forma geral dos modelos discretos estocásticos, com taxa de crescimento dependente da densidade e com intervenção é apresentada na equação (1), em que $N_{t}$ é o tamanho da população, $r\left(N_{t}\right)$, a taxa de crescimento da população e $0 \leq h_{t} \leq 1$, a taxa de $h_{t}$ abate em determinado tempo $t$.

$$
N_{t+1}=N_{t} \exp \left\{r\left(N_{t}\right)+\varepsilon_{t}\right\}-N_{t} h_{t}
$$

A variabilidade decorrente de fatores ambientais é representada pelo processo estocástico , o qual assumiu-se, neste estudo, seguir a distribuição Gaussiana com média zero e variância $\varepsilon_{t}$.

Adotou-se o modelo de Richards com intervenção (Loibel, 2004) por ser um modelo discreto estocástico resultante da generalização dos modelos logístico e de Gompertz e por considerar o abate de parte da população uma taxa denotada por $\tau^{-1}$.

A taxa de crescimento proposta por Richards (Richards, 1959) foi inicialmente utilizada para se estimar o crescimento do animal conforme a idade e, depois, para o crescimento de populações, sendo necessário apenas substituir na equação (1) a taxa de crescimento de Richards, descrita como:

$$
r\left(N_{t}\right)=\frac{\rho}{q}\left[1-\left(\frac{N_{t}}{K}\right)^{q}\right]
$$


Tabela 1- Número efetivo de animais e taxa de abate do rebanho bovino brasileiro no período de 1983 a 2000

Table 1 - Effective number of animals and slaughter rate of Brazilian cattle herd, in the period from 1983 to 2000

\begin{tabular}{lcc}
\hline $\begin{array}{c}\text { Ano } \\
\text { Year }\end{array}$ & $\begin{array}{c}\text { Número efetivo de animais } \\
\text { Effective number of animals }\end{array}$ & $\begin{array}{c}\text { Taxa de abate, } \% \\
\text { Slaughter rate, } \%\end{array}$ \\
\hline 1983 & 124798475 & 16,04 \\
1984 & 126528255 & 16,33 \\
1985 & 128362982 & 15,20 \\
1986 & 131870251 & 14,79 \\
1987 & 134084435 & 18,00 \\
1988 & 141360698 & 16,94 \\
1989 & 144442495 & 16,73 \\
1990 & 151719702 & 18,95 \\
1991 & 154297163 & 19,35 \\
1992 & 153033849 & 19,29 \\
1993 & 151419477 & 18,76 \\
1994 & 151839661 & 20,19 \\
1995 & 153776057 & 21,26 \\
1996 & 153320622 & 20,52 \\
1997 & 153647424 & 20,44 \\
1998 & 154677679 & 20,06 \\
1999 & 157447136 & 20,86 \\
2000 & 159401088 & 21,41 \\
\hline
\end{tabular}

Fonte: ANUALPEC (2001).

Source: ANUALPEC (2001).

O parâmetro $\rho$ representa a taxa de crescimento intrínseca à população e não depende de seu tamanho; é simplesmente a diferença entre as taxas de natalidade e a de mortalidade da população em questão: $\rho=\mathrm{d}-\mathrm{m}$. O parâmetro é a capacidade de suporte da população, ou seja, o tamanho limite a partir do qual a taxa de crescimento se torna decrescente. Esse nível é imposto pelo meio, basicamente por escassez de alimentos e de espaço.

O parâmetro $q$ representa um fator intrínseco à população. Quando se utiliza o modelo de Richards para estudos de curvas de crescimento, esse fator está relacionado ao metabolismo dos animais, o que facilita sua interpretação. No caso do crescimento populacional, esse parâmetro descreve a forma como a taxa diminui à medida que $N_{t}$ aumenta, isto é, se $q>1$ tem-se uma queda suave; se $q<1$, a queda é mais rápida e se $q=1$, a queda é linear. Em caso de variação do valor de $q$, tem-se uma infinidade de modelos.

Substituindo a equação (2) pela (1), tem-se o modelo de Richards com intervenção:

$$
N_{t+1}=N_{t} \exp \left\{\frac{\rho}{q}\left[1-\left(\frac{N_{t}}{K}\right)^{q}\right]+\varepsilon_{t}\right\}-h_{t} N_{t}
$$

Para se obterem inferências com o modelo de Richards em estudos de crescimento populacional, utiliza-se o método de máxima verossimilhança profile, pelo qual pode-se estimar os parâmetros de interesse, fixando-se um deles (Seber \& Wild, 1989).

Em alguns estudos de populações em se tem poucas observações, não é possível estimar a capacidade de suporte $(K)$, simplesmente porque a população não atingiu esse nível no período observado. Quando isso ocorre, pode-se obter informações sobre a capacidade de suporte da população na literatura e/ ou com especialistas na área.

Pode-se reescrever a equação (3) como uma relação linear considerando-se o parâmetro $q$ fixo e denotando-se $Y_{t}^{(q)}=\ln \left[\frac{N_{t+1}}{N_{t}}+h_{t}\right]^{q} ; X_{t}^{(q)}=\left(\frac{N_{t}}{K}\right)^{q}$ e $Z_{t}=q \varepsilon_{t}$. Assim, tem-se:

$$
Y_{t}^{(q)}=\rho\left[1-X_{t}^{(q)}\right]+Z_{t}
$$

Para obter-se a função de verossimilhança profile, necessita-se da densidade condicional de $N_{t+1}$ dado $N_{t}$ denotada por $P\left(N_{t+1} \mid N_{t}\right)$, que é obtida considerando-se resultados conhecidos da inferência estatística clássica (Bickel \& Doksum, 1977). O resumo desses resultados encontra-se a seguir e sua descrição detalhada pode ser consultada em Loibel (2004).

Na equação (4) $Z_{t}$ tem densidade Gaussiana com média zero e variância $\tau_{0}^{-1}=q^{2} \tau^{-1}$. Desta forma, a densidade condicional $P\left(Y_{t}^{(q)} \mid N_{t}^{(q)}\right)$, em que $N_{t}{ }^{(q)}=N_{t}{ }^{q}$ é Gaussiana com média $\alpha_{0}+\alpha_{1} N_{t}{ }^{(q)} \mathrm{e}$ variância $\tau_{0}^{-1}=q^{2} \tau^{-1}$.

A densidade de $P\left(N_{t+1} \mid N_{t}\right)$ pode ser escrita como:

$$
P\left(N_{t+1} \mid N_{t}\right)=P\left(Y_{t}^{(q)} \mid N_{t}^{(q)}\right) J_{Y N}
$$

em que $J_{Y N}=\frac{q}{N_{t+1}+h_{t} N_{t}}$ é o jacobiano da transformação.

Portanto, tem-se:

$$
P\left(N_{t+1} \mid N_{t}\right)=\left(\frac{\tau_{0}}{2 \pi}\right)^{1 / 2} \exp \left\{-\frac{\tau_{0}}{2}\left(Y_{t}^{(q)}-\rho\left[1-X_{t}^{(q)}\right]\right)^{2}\right\} \frac{q}{N_{t+1}+h_{t} N_{t}}
$$

A função de verossimilhança profile é representada por: 


$$
L\left(q, \alpha_{0}, \alpha_{1}, \tau_{0} \mid D\right)=\prod_{t=1}^{n-1} P\left(N_{t+1} \mid N_{t}\right)
$$

em que $n$ é o tamanho do conjunto de dados, denotado por $D$.

A partir da construção da função de verossimilhança (6) com o modelo (5), obtêm-se os estimadores de máxima verossimilhança profile dos parâmetros $\tau_{\mathrm{o}}$ e $\rho$ (Barndorff-Nielsen, 1994), descritos como:

$$
\begin{array}{r}
\hat{\tau}_{0}=\frac{n-1}{\sum_{t=1}^{n-1}\left(Y_{t}^{(q)}-\hat{\rho}\left[1-X_{t}^{(q)}\right]\right)^{2}} \\
\hat{\rho}=\frac{\sum_{t=1}^{n-1} Y_{t}^{(q)}\left[1-X_{t}^{(q)}\right]}{\sum_{t=1}^{n-1}\left[1-X_{t}^{(q)}\right]^{2}}
\end{array}
$$

As estimativas dos intervalos de confiança para os parâmetros $\tau_{\mathrm{o}}$ e $\rho$ são obtidas com base na propriedade de normalidade assintótica dos estimadores de máxima verossimilhança profile (7) e (8) e utilizando-se a matriz de informação de Fisher observada (Bickel \& Doksum, 1977), obtendo-se estimativas de desviospadrão, $d p\left(\hat{\tau}_{0}\right)$ e $d p(\hat{\rho})$. Os intervalos assintóticos com 100(1-a)\% de confiança para $\tau_{\mathrm{o}}$ e $\rho$ são representados por: $\hat{\tau}_{0} \pm z_{\alpha} d p\left(\hat{\tau}_{0}\right)$ e $\hat{\rho} \pm z_{\alpha} d p(\hat{\rho})$, em que $z_{\alpha}$ éo percentil da distribuição Gaussiana padrão.

Para o cálculo de probabilidades que envolvem dados correlacionados como os da Tabela 1, utiliza-se a modelagem do processo $N_{t}$ por cadeia de Markov (Çinlar, 1975), admitindo-se que a população de bovinos, no período 1983 a 2000, pode ser representada por um conjunto de $N_{I}$ intervalos ou "estados da cadeia", do tipo $I_{1}, I_{2}, \ldots ., I_{N_{I}}$. Assim, a probabilidade de o rebanho atingir determinado tamanho em tempo fixo é a probabilidade de determinado valor estar contido em um intervalo $I_{i}$.

Sejam $I_{i}$ e $I_{j}$ dois intervalos. Então, a probabilidade de transição do estado $I_{i}$ para o estado $I_{j}$ é:

$$
P_{i j}=P\left(N_{t+1} \in I_{j} \mid N_{t} \in I_{i}, 0<i, j \leq N_{I}, i \neq j\right)
$$

Seja a matriz $P$ com entradas $P_{i j}$ definidas para todo $\left\{(i, j), 0<i, j \leq N_{I}\right\}$. O interesse é calcular a probabilidade de a cadeia sair de um estado $I_{i}$ no instante $j$ e atingir determinado estado $I_{j}$ no instante $t+s, \quad s \geq 0$. Para isso, pode-se adotar a equação recursiva de Chapmann-Kolmogorov (Çinlar, 1975) para cálculo da matriz de probabilidade de transição em $s$ passos, denotada por $P^{s}$ :

$$
P^{s}=P^{s-t} P^{t}(9)
$$

Para um intervalo qualquer $I_{j}=\left(A_{j}, B_{j}\right)$, existe um intervalo $\left(a_{j}, b_{j}\right)$ associado a $\varepsilon_{t}$, de modo que a probabilidade $P_{i j}=P\left(a_{j} \leq \varepsilon_{t} \leq b_{j}\right)$. Assim, a matriz para o modelo (3) écalculada como: $P_{i j}=\phi\left(\tau^{1 / 2} B_{j}\right)-\phi\left(\tau^{1 / 2} A_{j}\right)$, em que $\phi$ é a função de distribuição acumulada gaussiana padrão.

Esta forma de se calcular a matriz de probabilidades de transição é uma aproximação discreta, uma vez são consideradas as probabilidades de transição entre os intervalos, não importando os valores contínuos que a população pode assumir dentro desses intervalos. O cálculo da probabilidade de se atingir determinada meta é feito assumindo-se a taxa de abate fixa durante o período estipulado.

Além da probabilidade de atingir determinada meta em tempo fixo, pode-se calcular também o tempo esperado para atingi-la. Nesses casos, são utilizados os resultados de cadeias de Markov, descritos por Heyman \& Sobel (1982). Em resumo, pode-se calcular esse tempo esperado resolvendo um sistema de equações. Sejam $N_{0} \in I_{i}$ e $\mu=\left(\mu_{i j}, 0 \leq i, j \leq N_{I}, i \neq j\right)$ os tempos esperados para se atingir o estado , partindo de qualquer estado $I_{i}$. Pode-se calcular $\mu$ pelo sistema de equações:

$$
(I-Q) \mu=1
$$

em que I é a matriz identidade de dimensão $\left(N_{I}-1\right)$, 1, um vetor de “uns” de dimensão $\left(N_{I}-1\right) \times 1$ e $Q$, a matriz de dimensão $\left(N_{I}-1\right)$, construída retirandose a linha e a coluna $j$ da matriz $P$.

\section{Resultados e Discussão}

Na Figura 1 é apresentada a estimava de crescimento da população e sua projeção para o período de 2001 a 2015. Utilizou-se o modelo de Richards e as estimativas da Tabela 2 . O crescimento do rebanho bovino foi irregular de 1983 a 2000, crescente de 1983 a 1991, apresentou oscilações de 1991 a 1995 e foi crescente daí por diante, o que significa que ainda não há dados suficientes para se estimar a capacidade de suporte dessa população. Optou-se, então, por estipular um valor alto para este nível (foi utilizado $K=400$ milhões, porém qualquer outro valor suficientemente 
Tabela 2 - Estimativas dos parâmetros do modelo de Richards com K = 400 milhões de animais, variabilidade decorrente dos fatores externos $\left(\tau^{-1}\right)$ taxa de crescimento $(\rho)$ e do parâmetro de forma $(q)$ e respectivos intervalos com $95 \%$ de confiança (IC)

Table 2 - Parameter estimates for Richards model with $K=$ 400 million of animals: variability due to external factors $\left(\tau^{-1}\right)$, growth rate $(\rho)$ and shape parameters (q) with respective $95 \%$ asymptotic confidence intervals (Cl)

\begin{tabular}{lcc}
\hline $\begin{array}{l}\text { Parâmetro } \\
\text { Parameter }\end{array}$ & $\begin{array}{c}\text { Estimativa } \\
\text { Estimate }\end{array}$ & $\begin{array}{c}\text { IC assintótico } \\
\text { Asymptotic CI }\end{array}$ \\
\hline$\tau$ & 3826 & {$[1200 ; 66451]$} \\
$q$ & 35,9000 & {$[2,2000 ; 37,6000]$} \\
$\rho$ & 6,5444 & {$[6,5365 ; 6,5220]$} \\
\hline
\end{tabular}

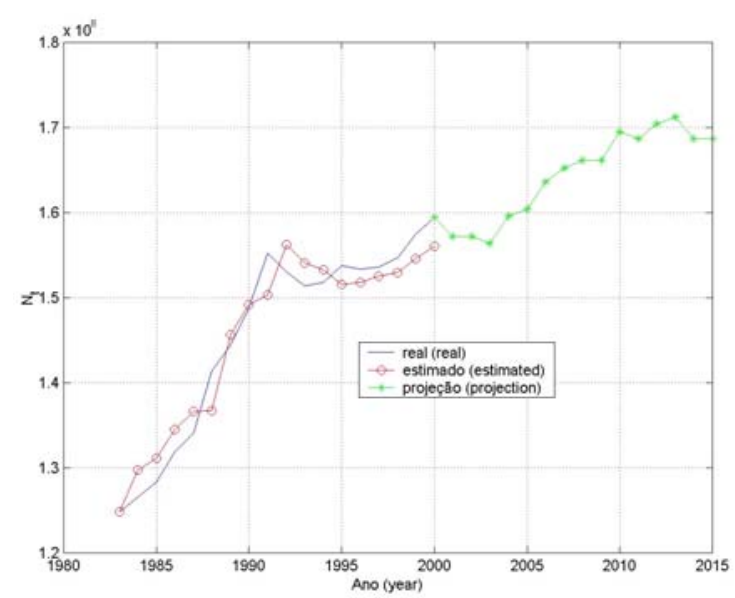

Figura 1 - Tamanho do rebanho bovino brasileiro: observado (-), estimado pelo modelo de Richards no período de 1983 a 2000 (- - - ) e projeção para o período de 2001 a 2015 ( * $_{*}$ ).

Figure 1 - Size of Brazilian cattle herd: observed (-), estimated by Richards model in the period of 1983 to 2000 ( $-\mathrm{-}-\mathrm{C})$ and projection for 2000 to 2015 period $(-*-)$.

grande, como por exemplo, 500 ou 600 milhões poderia ser usado) e estimar, então, por máxima verossimilhança profile, os parâmetros de interesse do modelo de Richards. Na Tabela 2 constam as estimativas de máxima verossimilhança dos parâmetros que representam a variabilidade decorrente de fatores externos à população $\left(\tau^{-1}\right)$, da taxa de natalidade $(\rho)$ e do parâmetro, para determinação da inclinação da curva de crescimento da população $(q)$. Estas estimativas proporcionaram taxa média de $18,23 \%$ de crescimento para o rebanho bovino.

Os valores da projeção de 2001 a 2015 (Figura 1) foram calculados considerando-se as taxas de abate constantes na Tabela 3. Os valores observados para a taxa de abate no período de 1983 a 2000 oscilaram de 14,79\% em 1986 a 21,41\% em 2000.

A projeção foi determinada efetuando-se a escolha de valores para a taxa de abate e simulação da oscilação na taxa do período anterior (1983 a 2000), para análise da forma pela qual esta taxa de abate afetaria o crescimento do rebanho. Como pode se observar na Tabela 3 e Figura 1, com essas taxas de abate, a população apresentaria crescimento lento, atingindo, no final do período (2015), pouco mais de 168 milhões de cabeças.

A escolha ideal para a taxa de abate seria aquela que proporcionasse máximo crescimento do rebanho e, ao mesmo tempo, atendesse satisfatoriamente à demanda. Para obtenção dessa taxa ótima de abate, é necessário o uso da técnica de programação dinâmica estocástica (Bertsekas, 2000, 2001).

Dessa forma, o cálculo da probabilidade de o rebanho bovino brasileiro atingir a meta de $200 \mathrm{mi}-$ lhões de animais até o ano de 2015 foi obtido aplicando-se um modelo de cadeia de Markov utilizando-se as estimativas pontuais clássicas e o "espaço de estados”, que é o conjunto de todos os tamanhos de população possíveis $E=\{124800000, \ldots, 220000000\}$.

Considerando-se os intervalos do espaço de estados descritos na Tabela 4, calculou-se a probabilidade de a cadeia sair do estado $N_{t}$, que é o número de animais observado no ano 2000 (Tabela 1), corres-

Tabela 3 - Projeção do crescimento da população bovina brasileira de 2001 a 2015 e taxa de abate, em porcentagem

Table 3 - Brazilian cattle herd growth projection for the years 2001 to 2015 and slaughter rate,\%

\begin{tabular}{lcc}
\hline $\begin{array}{c}\text { Ano } \\
\text { Year }\end{array}$ & $\begin{array}{c}\text { Tamanho da população } \\
\text { Population size }\end{array}$ & $\begin{array}{c}\text { Taxa de abate, } \% \\
\text { Slaughter rate, } \%\end{array}$ \\
\hline 2001 & 157170000 & 20,00 \\
2002 & 157190000 & 20,50 \\
2003 & 156430000 & 18,00 \\
2004 & 159580000 & 19,50 \\
2005 & 160390000 & 18,00 \\
2006 & 163620000 & 19,00 \\
2007 & 165280000 & 19,50 \\
2008 & 166120000 & 20,00 \\
2009 & 166150000 & 18,00 \\
2010 & 169490000 & 20,50 \\
2011 & 168660000 & 19,00 \\
2012 & 170370000 & 19,50 \\
2013 & 171240000 & 21,50 \\
2014 & 168700000 & 20,00 \\
2015 & 168720000 & 19,50 \\
\hline
\end{tabular}


pondente ao intervalo $I_{15}$, e atingir em 15 anos o estado $N_{t}=200$ milhões, que corresponde ao intervalo $I_{32}$. O cálculo dessa probabilidade foi feito com base em alguns valores fixos para a taxa de abate.

Considerando-se equação recursiva de ChapmanKolmogorov (9) e as entradas $P\left(N_{15} \in I_{j} \mid N_{1} \in I_{i}\right)$, com $i, j=1, \ldots, 40$, a matriz $P^{15}$ foi calculada para várias taxas de abate, de modo que a probabilidade $P\left(N_{15} \geq 200 \times 10^{6} \mid N_{1} \in I_{15}\right)$ é representada por:

$P\left(N_{15} \geq 200 \times 10^{6} \mid N_{1} \in I_{15}\right)=\sum_{j=32}^{40}\left(N_{15} \in I_{j} \mid N_{1} \in I_{15}\right)$

Na Figura 2 constam as probabilidades de o rebanho atingir 200 milhões de cabeças em 2015 considerando-se diversos valores da taxa de abate entre 16 e $20 \%$.

Pineda (2000) afirma que a taxa de abate do rebanho brasileiro, que em 1980 era de $15 \%$, passou a ser de aproximadamente $22 \%$ em 2000 . Segundo o autor, se esta tendência de crescimento se mantiver, a população bovina brasileira atingirá 200 milhões de animais em 2015, com abate de $40 \%$. Os resultados obtidos neste trabalho não confirmam esta hipótese, pois a taxa média de crescimento observada foi de $18,23 \%$ e qualquer taxa de abate fixa acima deste valor resultaria em diminuição da população de bovinos, e não em aumento. A probabilidade de o rebanho brasileiro atingir 200 milhões de animais em 2015, com taxa anual de abate constante e acima de 19\%, é praticamente nula, ou seja, $\mathrm{P}(15)=0,00081$ (Figura 2). A maior probabilidade seria $\mathrm{P}(15)=0,9853$, obtida com taxa de abate fixa de $16 \%$, que é muito baixa.

Além do cálculo da probabilidade de o rebanho atingir 200 milhões de cabeças em 2015, também foi calculado o tempo para se atingir esse valor, obtido com a solução do sistema de equações (10) para alguns valores da taxa de abate. Na Figura 3 constam os tempos em função da taxa de abate. Observa-se

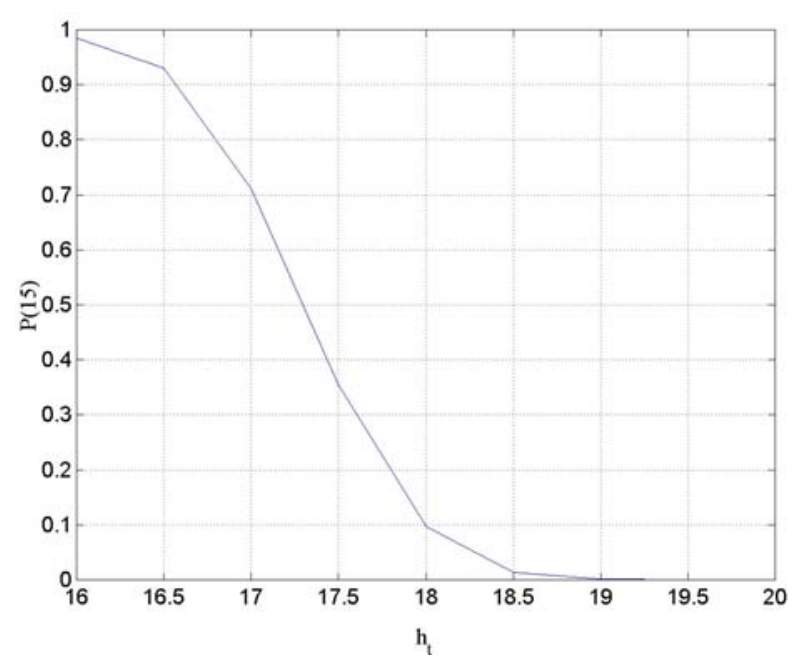

Figura 2 - Probabilidades de o rebanho bovino brasileiro atingir 200 milhões de animais no ano de 2015, em função das taxas anuais de abate

Figure 2 - Probabilities of the Brazilian cattle herd reaching 200 millions animals in 2015, as a function of the annual slaughter rate

Tabela 4 - Intervalos do espaço-estados para o cálculo da probabilidade de o rebanho bovino brasileiro passar de 159 milhões $\left(I_{15}\right)$ para 200 milhões de animais $\left(I_{32}\right)$

Table 4 - Intervals of the space-states to calculate the probability of Brazilian cattle herd to increase from 159 millions $\left(I_{15}\right)$ to 200 millions of animals $\left(I_{32}\right)$

\begin{tabular}{lccc}
\hline $\mathrm{I}_{1}$ & $<124800000$ & $\mathrm{I}_{21}$ & {$[172400000 ; 174900000]$} \\
$\mathrm{I}_{2}$ & {$[124800000 ; 127300000]$} & $\mathrm{I}_{22}$ & {$[174900000 ; 177410000]$} \\
$\mathrm{I}_{3}$ & {$[127300000 ; 129810000]$} & $\mathrm{I}_{23}$ & {$[177410000 ; 179920000]$} \\
$\mathrm{I}_{4}$ & {$[129810000 ; 132310000]$} & $\mathrm{I}_{24}$ & {$[179920000 ; 182420000]$} \\
$\mathrm{I}_{5}$ & {$[132310000 ; 134820000]$} & $\mathrm{I}_{25}$ & {$[182420000 ; 184930000]$} \\
$\mathrm{I}_{6}$ & {$[134820000 ; 137320000]$} & $\mathrm{I}_{26}$ & {$[184930000 ; 187430000]$} \\
$\mathrm{I}_{7}$ & {$[137320000 ; 139830000]$} & $\mathrm{I}_{27}$ & {$[187430000 ; 189940000]$} \\
$\mathrm{I}_{8}$ & {$[139830000 ; 142340000]$} & $\mathrm{I}_{28}$ & {$[189940000 ; 192440000]$} \\
$\mathrm{I}_{9}$ & {$[142340000 ; 144840000]$} & $\mathrm{I}_{29}$ & {$[192440000 ; 194950000]$} \\
$\mathrm{I}_{10}$ & {$[144840000 ; 147350000]$} & $\mathrm{I}_{30}$ & {$[194950000 ; 197450000]$} \\
$\mathrm{I}_{11}$ & {$[147350000 ; 149850000]$} & $\mathrm{I}_{31}$ & {$[197450000 ; 199960000]$} \\
$\mathrm{I}_{12}$ & {$[149850000 ; 152360000]$} & $\mathrm{I}_{32}$ & {$[199960000 ; 202460000]$} \\
$\mathrm{I}_{13}$ & {$[152360000 ; 154860000]$} & $\mathrm{I}_{33}$ & {$[202460000 ; 204970000]$} \\
$\mathrm{I}_{14}$ & {$[154860000 ; 157370000]$} & $\mathrm{I}_{34}$ & {$[204970000 ; 207470000]$} \\
$\mathrm{I}_{15}$ & {$[157370000 ; 159870000]$} & $\mathrm{I}_{35}$ & {$[207470000 ; 209980000]$} \\
$\mathrm{I}_{16}$ & {$[159870000 ; 162380000]$} & $\mathrm{I}_{36}$ & {$[209980000 ; 212480000]$} \\
$\mathrm{I}_{17}$ & {$[162380000 ; 164880000]$} & $\mathrm{I}_{37}$ & {$[212480000 ; 214990000]$} \\
$\mathrm{I}_{18}$ & {$[164880000 ; 167390000]$} & $\mathrm{I}_{38}$ & {$[214990000 ; 217490000]$} \\
$\mathrm{I}_{19}$ & {$[167390000 ; 169890000]$} & $\mathrm{I}_{39}$ & {$[217490000 ; 220000000]$} \\
$\mathrm{I}_{20}$ & {$[169890000 ; 172400000]$} & $\mathrm{I}_{40}$ & $\mathrm{~N}_{\mathrm{t}}>220000000$ \\
\hline
\end{tabular}


que, com taxa de abate de $16 \%$, seria possível atingir, em apenas 11 anos, 200 milhões de animais. Contudo, para taxa de abate fixa de 19\%, o tempo necessário seria de 42 anos.

Os resultados apresentados neste trabalho foram obtidos a partir de estimativas e predições utilizando-se um modelo estatístico considerando-se o número efetivo de animais e a taxa de abate do rebanho brasileiro no período de 1983 a 2000. Entretanto, deve-se salientar que a projeção de crescimento do rebanho bovino brasileiro pelo modelo de Richards não permite incorporar mudanças decorrentes de fatores de ações estritamente zootécnicas, como nutrição, melhoramento genético, sanidade, manejo, entre outras. Com a globalização, a atividade pecuária ainda é fortemente dependente de fatores como política interna e de exportação de carne bovina, campanhas de marketing, entre outros.

Contudo, as inferências a partir modelo de Richards comprovam que é possível predizer a evolução do rebanho bovino brasileiro, a fim de se obter uma situação ideal para tamanho do rebanho, taxa de abate e número de anos para atingir determinada meta.

É possível ainda obter respostas para perguntas como: Qual taxa de abate deve ser utilizada para o rebanho atingir determinado tamanho em determinado ano? Quais valores resultam em maiores probabilidades de esta meta ser atingida? Com que taxa de abate o rebanho atinge determinado tamanho em menor intervalo de tempo? Deve-se, no entanto, incentivar pesquisas visando ao uso de técnicas de programação dinâmica estocástica e desenvolver modelos de

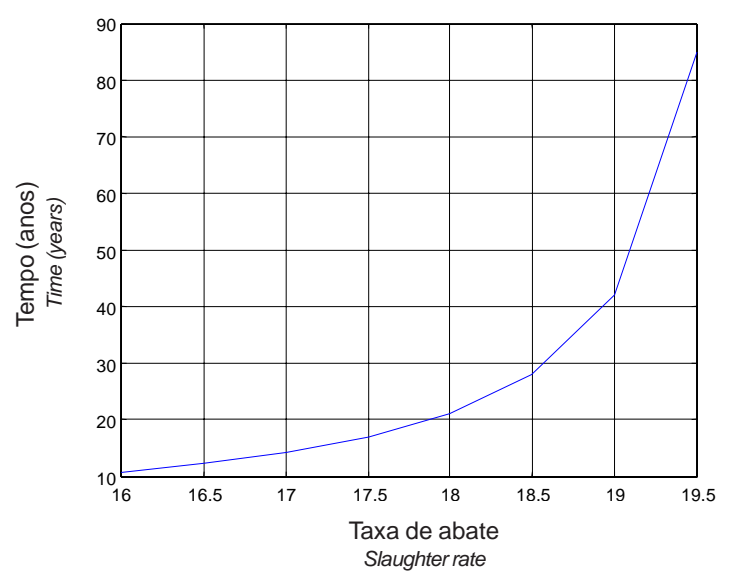

Figura 3 - Tempo, em anos, esperado para o rebanho bovino atingir 200 milhões de animais em função da taxa de abate.

Figure 3 - Expected time, in years, for the Brazilian cattle herd to reach 200 million animals. otimização que possibilitem determinar uma política ótima de abate para garantia de taxa de abate crescente em uma população também crescente.

O Brasil pode se consolidar definitivamente com uma população bovina de aproximadamente $200 \mathrm{mi}-$ lhões de animais e se fortalecer como fornecedor mundial de carnes, com reflexos positivos na captação de divisas para o país. Além do potencial de incremento de consumo de carne bovina no mercado interno, um aspecto de extrema importância e que influencia diretamente os sistemas produtivos é a sustentabilidade.

Quanto ao mercado externo, é importante ressaltar as exigências de controle ambiental impostas pelos países mais desenvolvidos, que se traduzem em padrões de requerimentos semelhantes para as importações. Nesse contexto, torna-se fundamental, entre outros fatores, que se atendam às exigências sanitárias, envolvendo tanto a saúde do rebanho como a saúde pública.

\section{Conclusões}

O ajuste do modelo de Richards utilizando-se o método da máxima verossimilhança profile se mostrou adequado para estimação do crescimento da população de bovina brasileira no período de 1983 a 2000.

A estimativa da taxa de crescimento global anual do rebanho bovino brasileiro é de $18,23 \%$, de modo que, com qualquer taxa de abate inferior a esse valor, a população continuará crescente.

Com probabilidade superior a 0,7 , estima-se que o rebanho bovino brasileiro atingia 200 milhões de animais em 11 anos, com taxa de abate anual de 16 a $17 \%$, e, em 20 anos, com taxa de abate de aproximadamente $18 \%$.

Pelo modelo de Richards é possível predizer a evolução do rebanho bovino brasileiro e encontrar uma situação ideal para tamanho do rebanho, taxa de abate e número de anos para se atingir determinada meta.

\section{Literatura Citada}

ANUALPEC 2001: Anuário da Pecuária Brasileira. São Paulo: FNP, 2001. 359p.

ANUALPEC 2002: Anuário da Pecuária Brasileira. São Paulo: FNP, 2002. 400p.

ANUALPEC 2003: Anuário da Pecuária Brasileira. São Paulo: FNP, 2003. 400p.

BARNDORFF-NIELSEN, O.E.; COX, D.R. Inference and asymptotics. London: Chapman \& Hall, 1994. 360p. 
BERTSEKAS, D.P. Dynamic programming and optimal control. 2.ed. Englewood Cliffs: Athena Scientific, 2001. v.2, 320p.

BERTSEKAS, D.P. Dynamic programming and optimal control. 2.ed. Englewood Cliffs: Athena Scientific, 2000. v.1, 520p.

BICKEL, P.J.; DOKSUM, K.A. Mathematical statistics. San Francisco: Holden-Day, 1977. 492p.

BOX, G.E.; COX, D.R. An analysis of transformations. Journal of the Royal Statistic Society, serie B, v.6, p.211-252, 1964.

ÇINLAR, E. Introduction to stochastic processes. New Jersey: Prentice-Hall, 1975. 402p.

FITZHUGH JR., H.A. Analysis of growth curves and strategies for altering their shape. Journal of Animal Science, v.42, n.4, p.1036-1051, 1976.

FREITAS, A.R.; ALBINO, L.F.; MICHELAN FILHO, T. et al. Modelos de curvas de crescimento em frangos de corte. Pesquisa Agropecuária Brasileira, v.19, n.9, p.1057-1064, 1984.

HAMMERLE, A.; ROQUE-SPECHT.V.; MOMBACH, J.C.M. et al. Predição da curva de crescimento de Escherichia coli utilizando modelos matemáticos em diferentes condições de temperatura e pH. http://www.inf.unisinos.br/ lbbc/ CBCTA2002.PDF (05/12/2002)

HEYMAN, D.P.; SOBEL, M.J. Stochastic models in operations research: stochastic processes and operating characteristics. New York: McGraw-Hill Book Company, 1982. v.1. 548p.

LOIBEL, S.M.C. Inferência e indicadores de viabilidade para modelos estocásticos de crescimento de populações. Campinas: Universidade Estadual de Campinas, 2004. 114p. Tese (Doutorado em Engenharia Elétrica) - Universidade Estadual de Campinas.

LUDWIG, D. Uncertainty and the assessment of extinction probabilities. Ecological Applications, v.6, n.4, p.10671076, 1996.
LUDWIG, A. Stochastic differential equations: theory and application. New York: John Wiley \& Sons, 1974. 45p.

PAZ, C.C.P. Associação entre polimorfismos genéticos e parâmetros da curva de crescimento em bovinos de corte. Piracicaba: Escola Superior de Agricultura Luiz de Queiroz, 2002. 107p. Tese (Doutorado em Melhoramento Animal) Escola Superior de Agricultura Luiz de Queiroz, 2002.

PEROTTO, D.; CUE, R.I.; LEE, A.J. Comparison of nonlinear functions for describing the growth curve of three genotypes of dairy cattle. Canadian Journal of Animal Science, v.72, n.3, p.773-782, 1992.

PINEDA, N.R. Cenário de cadeia produtiva da carne bovina no Brasil. ABCZ, v.2, n.10, p.186-187, 2002.

PINEDA, N.R. Influência do Nelore na produção de carne no Brasil. In: SIMPÓSIO NELORE, 2000, Ribeirão Preto. Anais... Ribeirão Preto: Associação Brasileira de Criadores de Nelore, 2000. p.3-13

RICHARDS, J.F. A flexible growth function for empirical use. Journal of Experimental Botanica, v.10, n.29, p.290-300, 1959.

SANDLAND, R.L.; McGILCHRIST, C.A. Stochastic growth curve analysis. Biometrics, v.35, n.1, p.255-271, 1979.

SEBER, G.A.F.; WILD, C.J. Nonlinear regression. New York: John Wiley, 1989. 768p.

Recebido em: 06/10/03 Aceito em: 07/07/05 